\title{
Evaluación y Comparación de la Estabilidad de Implantes Dentales en el Maxilar y la Mandíbula en Tres Tiempos Distintos, Mediante Análisis de Frecuencia de Resonancia
}

\author{
Comparison of Dental Implants Stability in Maxilla and Mandible \\ at Three Different Times Using Resonance Frequency Analysis
}

\author{
Osvaldo Gahona Gutiérrez "**; Xenia Granic Marinov"; María Cristina Antúnez Chelmes"*; \\ Juan Argandoña Pozo ${ }^{*+* *}$; Matteo de la Fuente Ávila"; Stefan Domancic Alucema"* \& Víctor Díaz-Narváez
}

GAHONA, G. O.; GRANIC, M. X.; ANTÚNEZ, CH. M. C.; ARGANDOÑA, P. J.; DE LA FUENTE, Á. M.; DOMANCIC, A. S. \& DÍAZ-NARVÁEZ, V. Evaluación y comparación de la estabilidad de implantes dentales en el maxilar y la mandíbula en tres tiempos distintos, mediante análisis de frecuencia de resonancia. Int. J. Odontostomat., 10(3):475-481, 2016.

RESUMEN: El propósito de este estudio fue medir y comparar la estabilidad de implantes dentales en maxilar y mandíbula en tres tiempos distintos, mediante análisis de resonancia de frecuencia (RFA). Mediante un diseño no experimental prospectivo, fueron evaluados 103 Implantes 3i Osseotite en 57 pacientes (50 en el maxilar y 53 en mandíbula). Mediante un dispositivo electrónico (Osstell-Mentor ${ }^{\circledR}$ ) se registró el coeficiente de estabilidad para cada implante (ISQ) al momento de la cirugía de implantes, a las 6 semanas y a las 8 semanas siguientes. Respecto al maxilar, las medianas aumentan progresivamente después de la cirugía de implantes. En la mandíbula, parecen ser similares entre la primera y la tercera medición, pero decrecen en la segunda. Independiente tanto para el maxilar como para la mandíbula, los valores de las medianas fueron altamente significativas, lo cual indica que estas difieren en los tres tiempos estudiados $(p<0,001)$. Se encontró diferencia significativa entre las medianas del maxilar y la mandíbula solamente al momento de la colocación de implantes. En los demás tiempos evaluados, no se observaron diferencias significativas $(p>0,05)$. En el maxilar, la estabilidad de los implantes aumenta con el tiempo. En la mandíbula, disminuye en la sexta semana y aumenta en las 8 siguientes, alcanzando valores similares a los iniciales. La estabilidad de los implantes es mayor en la mandíbula que en el maxilar, solo al momento de la cirugía. A las 8 semanas, la estabilidad alcanzada por los implantes en el maxilar y la mandíbula son similares.

PALABRAS CLAVE: implantes dentales, maxilar, mandíbula, análisis de frecuencia de resonancia.

\section{INTRODUCCIÓN}

Los implantes dentales, requieren de una correcta oseointegración para una rehabilitación estética y funcional perdurable. El maxilar y la mandíbula, por las funciones que realiza la musculatura adherida a ellos, son estructuralmente distintos respecto a su componente cortical, trabecular y vascular, factores que junto a otros, inciden en el proceso de oseointegración. Por esto, podría ser esperable que éste proceso varíe entre uno y otro (Davies, 2003). Determinar la estabilidad de los implantes en el tejido óseo, es un factor clave para saber cuándo someter- los a carga e iniciar el proceso de rehabilitación, sin afectarla.

La estabilidad de los implantes se divide en dos fases. Inicialmente, la estabilidad primaria que es netamente mecánica, ya que corresponde a la resistencia y rigidez de la unión hueso implante por la presión de este, al momento de su inserción, determinando si es posible o no someterlo a carga. Posteriormente ocurre la estabilidad secundaria, también llamada estabilidad biológica, donde se produce la for-

\footnotetext{
Facultad de Odontología, Universidad Finis Terrae, Chile.

* Departamento de Cirugía y Traumatología Máxilo-Facial, Facultad de Odontología, Universidad de Chile.

*** Escuela de Postgrado. Facultad de Odontología, Universidad San Sebastián.

**** Servicio de Cirugía Bucal y Maxilofacial. Hospital San Juan de Dios, Santiago de Chile.

*.*m** Facultad de Odontología, Universidad San Sebastián, Chile.

********universidad Autónoma de Chile, Chile.
} 
GAHONA, G. O.; GRANIC, M. X.; ANTÚNEZ, CH. M. C.; ARGANDOÑA, P. J.; DE LA FUENTE, Á. M.; DOMANCIC, A. S. \& DÍAZ-NARVÁEZ, V. Evaluación y comparación de la estabilidad de implantes dentales en el maxilar y la mandíbula en tres tiempos distintos, mediante análisis de frecuencia de resonancia. Int. J. Odontostomat., 10(3):475-481, 2016.

mación de hueso en contacto directo con la superficie del implante (Atsumi et al., 2007).

Al momento de la cirugía de implantes, la estabilidad primaria presenta el $100 \%$ de su estabilidad, mientras que la estabilidad secundaria el $0 \%$. La estabilidad primaria y la secundaria son inversamente proporcionales, alrededor de la tercera semana se cruzan alcanzando ambas aproximadamente el $30 \%$. Se estabilizan a las 8 semanas post-quirúrgicas, cuando la estabilidad primaria empieza a tender a $0 \%$ y la secundaria al $80 \%$ producto de la oseointegración (Raghavendra et al., 2005).

Existen distintos métodos para cuantificar la estabilidad de los implantes oseointegrados. Métodos invasivos como los histológicos y microscópicos, que representan el Gold Standard, pero carecen de reproductibilidad clínica, y métodos no invasivos como el torque de inserción, el torque de remoción y la respuesta a la percusión. En este último se basa el Periotest y el análisis de frecuencia de resonancia, en inglés Resonance Frequency Analysis, RFA (Mihoko et al.).

El análisis de frecuencia de resonancia es una prueba para evaluar la estabilidad de implantes mediante la medición de la frecuencia de la oscilación de este, dentro del tejido óseo. El implante es sometido a una ligera fuerza lateral, que ocasiona un desplazamiento lateral del implante debido a la deformación elástica del hueso (Meredith et al., 1995; Meredith et al., 1996; Degidi et al., 2010). Desde hace más de una década, este método se ha utilizado por ser confiable, predecible y objetivo para medir la estabilidad de los implantes oseointegrados, para determinar los efectos de la carga inmediata o temprana y para evaluar los cambios que ocurren durante el periodo de oseointegración (Degidi et al.; Koh et al., 2010 \& Friberg et al., 1999).

La medición se efectúa mediante una barra de metal Ilamada Smartpeg (similar a un pilar protésico) que se atornilla directamente al implante, que actúa como transductor. En su porción superior, posee un imán que es excitado por impulsos magnéticos producidos por el mismo aparato de medición. Esta frecuencia de resonancia se expresa de forma electromagnética como un cociente de estabilidad del implante (Implant stability Quocient, ISQ) con unidades que van desde 1 a 100, donde valores bajos indican inestabilidad y valores altos mayor estabilidad. Este valor representa la rigidez de la interfase implante-hueso. Según el fabricante, un implante exitoso, se asocia a valores ISQ mayores a 65 , mientras que implantes con valores ISQ menores a
50, indican alto riesgo de falla. (Meredith et al., 1996; Herrero-Climent et al., 2012).

El Osstell mentor ${ }^{\circledR}$ tercera generación, es una de las últimas versiones de este instrumento. No requiere de un computador para completar su análisis y al ser rápido y fácil de aplicar, permite ser utilizado a diario en la práctica clínica (Herrero-Climent et al.) El objetivo de este estudio fue evaluar y comparar la estabilidad de los implantes oseointegrados mediante el uso del análisis de frecuencia de resonancia, tanto en el maxilar y la mandíbula, como entre ellos, en un periodo de 2 meses.

\section{MATERIAL Y MÉTODO}

Selección de pacientes (criterios de admisión). Fueron seleccionados 90 pacientes con necesidad de 159 implantes, de entre todos los que acudieron a los postgrados de Cirugía Bucal y Rehabilitación Oral de la Facultad de Odontología de la Universidad Finis Terrae entre los años 2014 y 2015. La evaluación preoperatoria incluyó examen clínico, radiografía panorámica y $\mathrm{CBCT}$ de la zona a intervenir.

\section{Criterios de inclusión:}

- Hombres y mujeres mayores de 18 años.

- Pacientes con necesidad de rehabilitación sobre implantes de uno o dos dientes.

- Físicamente disponibles para tolerar procedimiento quirúrgico convencional y rehabilitador.

Los criterios de exclusión fueron:

- Pacientes con infección y/o inflamación en el sitio quirúrgico.

- Pacientes fumadores pesados, que consuman más de 10 cigarrillos al día.

- Pacientes con Diabetes Mellitus no controlada.

- Pacientes con patología metabólica ósea como: Osteomalacia, hiperparatiroidismo primario o secundario, osteodistrofia renal o enfermedad de Paget.

- Pacientes con historia terapéutica de radiación de cabeza y cuello.

- Paciente con necesidad de injerto óseo en el sitio destinado a la rehabilitación mediante implantes.

- Pacientes que no tienen certeza de no estar embarazadas al momento de la evaluación pre-quirúrgica.

- Pacientes con evidencia de para-funciones como bruxismo o apriete dentario. 
GAHONA, G. O.; GRANIC, M. X.; ANTÚNEZ, CH. M. C.; ARGANDOÑA, P. J.; DE LA FUENTE, Á. M.; DOMANCIC, A. S. \& DÍAZ-NARVÁEZ, V. Evaluación y comparación de la estabilidad de implantes dentales en el maxilar y la mandibula en tres tiempos distintos, mediante análisis de frecuencia de resonancia. Int. J. Odontostomat., 10(3):475-481, 2016.

Todos firmaron un consentimiento para participar en el estudio y fueron informados, según norma la declaración de Helsinki. En aquellos pacientes que requerían exodoncia de dientes para posteriormente rehabilitar mediante implantes, fue necesario un período de cicatrización de 3 meses. Solo 57 pacientes completaron el estudio, quedando un total de 103 implantes, 50 instalados en el maxilar y 53 instalados en la mandíbula.

Procedimiento y protocolo de medición de ISQ. Se utilizaron implantes 3i Osseotite paralelos con diámetros de 4,0 y 5,0 mm. y de longitudes de 10, 11,5 y $13 \mathrm{~mm}$. Los que fueron posicionados por 2 operadores calibrados especialistas en Implantología Oral, según norma la secuencia de fresado para dichos implantes del fabricante. En todos los casos se realizó el acceso mediante un colgajo de espesor total exponiendo la zona a intervenir. Todos los implantes fueron posicionados en un solo tiempo quirúrgico ( 1 o 2 implantes según el caso). Pacientes con necesidad de injerto óseo no fueron considerados para el estudio, pero en aquellos casos que durante la cirugía se evidenció socavados laterales que necesiten ser regenerados después de la instalación del implante, fue aceptable agregar hueso particulado y no excluyó al paciente del estudio. Todos los implantes quedaron en posición epicrestal.

El procedimiento se dividió en 3 etapas:

1) Etapa 1: Al momento de la cirugía (día 0). Fresado del lecho quirúrgico e instalación del implante Se atornilló el SmartPeg y se procedió a realizar la medición del ISQ con un equipo de RFA Osstell mentor ${ }^{\circledR} 3$ ra generación, según el manual del fabricante, para la primera medición de la estabilidad del implante. Todos los implantes quedaron conectados a boca mediante un pilar de cicatrización.

2) Etapa 2: A las 6 semanas desde la cirugía de implantes, se procedió a retirar el pilar de cicatrización y atornillar un nuevo SmartPeg para obtener la segunda medición del ISQ del implante. Posteriormente se volvió a atornillar el pilar de cicatrización.

3) Etapa 3: A los 2 meses desde la cirugía de implantes, se procedió nuevamente a retirar el pilar de cicatrización y atornillar un nuevo SmartPeg para obtener la tercera medición del ISQ del implante. Luego se atornilló una corona provisoria en espera del tiempo necesario para la rehabilitación definitiva, según protocolo convencional.
Análisis estadístico. Mediante un diseño no experimental prospectivo, todos los valores ISQ obtenidos tanto en los implantes del maxilar y la mandíbula, fueron sometidos inicialmente a la prueba de Bondad de Ajuste de Kolmogorov-Smirnov (K-S) con la corrección de Lilliefors para la significación (Siegel et al., 1998). Y la prueba de homocedasticidad de Levene (Díaz, 2009a; 2009b). Posteriormente se estimaron la mediana y los cuartiles en cada uno de las mediciones realizadas y la mediana general de los datos. Las medianas de las tres mediciones tanto en el maxilar como para la mandíbula, fueron comparados entre sí mediante la prueba de Friedman para k muestras dependientes (Díaz, 2009a; 2009b). La comparación entre la distribución de los datos de los valores ISQ estudiados en el maxilar y en la mandíbula se realizó mediante la prueba de KolmogorovSmirnov para dos muestras independientes (Díaz, 2009a; 2009b). Se estimó la significación asintótica y, con el objeto de aumentar la precisión de la estimación de diferencias, se empleó también la significación exacta. Las medianas fueron mostradas, además, en gráficos de cajas. El nivel de significación empleado en todos los casos fue de $a \leq 0,05$.

\section{RESULTADOS}

En la Tabla I, se presentan los resultados de la aplicación de la prueba de bondad de ajuste de K-S con la corrección antes descrita a los datos de cada unode las mediciones y tiempos estudiados (ISQ en el maxilar y la mandíbula). Se observaron diferencias significativas $(p<0,05)$ y altamente significativas en $(p<0,005)$, pero los datos de algunos de los tratamientos no fueron significativos $(p>0,05)$ todo lo cual permite inferir que algunos de los datos de los tratamientos examinados no tienen distribución normal.

En la Tabla II, se muestran los resultados de la comparación de varianzas entre los valores ISQ basados en la media y en la mediana tanto para el maxilar, así como para la mandíbula. En todos casos la prueba de Levene fue altamente significativa $(p<0,001)$, lo que demuestra que no existe homocedasticidad entre los valores ISQ en el maxilar, así como en la mandíbula. Estos resultados obligan a seguir tratando los datos con técnicas no paramétricas (Díaz, 2009a; 2009b).

En la Tabla III, se muestran los resultados de las medianas y los percentiles en cada uno de los tres tiempos donde se registró el valor ISQ de los implantes en el maxilar. En el gráfico de cajas (Fig. 1) se observa 
GAHONA, G. O.; GRANIC, M. X.; ANTÚNEZ, CH. M. C.; ARGANDOÑA, P. J.; DE LA FUENTE, Á. M.; DOMANCIC, A. S. \& DÍAZ-NARVÁEZ, V. Evaluación y comparación de la estabilidad de implantes dentales en el maxilar y la mandíbula en tres tiempos distintos, mediante análisis de frecuencia de resonancia. Int. J. Odontostomat., 10(3):475-481, 2016.

Tabla I. Resultados de la aplicación de los prueba de normalidad a los datos de cada tratamiento examinado.

\begin{tabular}{llccr}
\hline & TRATAMIENTOS & \multicolumn{2}{c}{ Kolmogorov-Smirnov } \\
& & Estadístico & gl. & Sig. \\
\hline \multirow{2}{*}{$\begin{array}{llll}\text { Datos Observados } \\
\text { en el Maxilar }\end{array}$} & ISQ al momento de la cirugía. &, 177 & 50 &, 0001 \\
& ISQ a las 6 semanas &, 142 & 50 & 0,013 \\
Datos Observados & ISQ a los 2 meses &, 118 & 50 & 0,078 \\
en la Mandibula & ISQ al momento de la cirugía &, 140 & 53 & 0,011 \\
& ISQ a las 6 semanas &, 080 & 53 & 0,200 \\
& ISQ a los 2 meses. &, 113 & 53 & 0,087 \\
\hline
\end{tabular}

Tabla II. Resultados de la aplicación de los prueba de homocedasticidad a los datos de cada tratamiento examinado.

\begin{tabular}{llcr}
\hline & & $\begin{array}{c}\text { Estadístico de } \\
\text { Levene }\end{array}$ & Sig. \\
\hline Datos Observados & Basándose en la media & 15,580 & 0,0002 \\
Maxilar & Basándose en la mediana. & 13,255 &, 0002 \\
& & & \\
Datos Observados & Basándose en la media & 7,001 & 0,0020 \\
Mandíbula & Basándose en la mediana. & 6,103 & 0,0030 \\
\hline
\end{tabular}

Tabla III. Resultados de la estimación de la mediana y los cuartiles de los valores ISQ en el maxilar en los tres tiempos examinados.

\begin{tabular}{lcccc}
\hline \multicolumn{1}{c}{ MAXILAR } & & & Percentiles & \\
& $\mathrm{N}$ & 25 & 50 (Mediana) & 75 \\
\hline ISQ al momento de la cirugía & 50 & 58,00 & 67,00 & 73,00 \\
ISQ a las 6 semanas. & 50 & 60,50 & 67,00 & 71,00 \\
ISQ a los 2 meses. & 50 & 68,75 & 71,50 & 75,00 \\
\hline
\end{tabular}

que los valores de las medianas aumentan sostenidamente y de acuerdo al orden de cómo fueron registrados los valores ISQ de los implantes. La comparación entre las medianas fue altamente significativa $(p<0,001)$, lo cual indica que estas difieren en los tiempos estudiados.

En la Tabla IV, se muestran los resultados de las medianas y los percentiles en cada uno de los tres tiempos en los cuales se realizó la medición de ISQ en los implantes en la mandíbula. En el gráfico de cajas (Fig. 2) se observa que la mediana es similar en el primer y tercer tiempo, pero disminuye en el segundo. La comparación entre las medianas fue altamente significativa $(p<0,001)$, lo cual indica que estas difieren en los tiempos estudiados.
Por último, en la Tabla $V$ se muestran los resultados de la comparación entre las distribuciones de las mediciones ISQ de los implantes instalados en el maxilar y la mandíbula, considerando en cada uno de ellos los tres tiempos obtenidos. La prueba de Chicuadrado fue significativa $(p<0,05)$ en la asíntótica, pero altamente significativa en la exacta $(p<0,01)$, lo cual indica que los datos considerados en los tres tiempos en cada una de las mediciones (ISQ en el maxilar y en la mandíbula) son diferentes entre sí, solamente al momento de la cirugía. A las 6 y 8 semanas, no existen diferencias significativas entre las distribuciones de los valores ISQ obtenidos para el maxilar y la mandíbula.

Tabla IV. Resultados de la estimación de la mediana y los cuartiles de los valores ISQ en la mandíbula en los tres tiempos examinados.

\begin{tabular}{lcccc}
\hline \multicolumn{1}{c}{ MANDIBULA } & N & 25 & Percentiles & (Mediana) \\
\hline ISQ al momento de la cirugía. & 53 & 66,00 & 72,00 & 75,00 \\
ISQ a las 6 semanas. & 53 & 61,50 & 66,00 & 72,00 \\
ISQ a los 2 meses. & 53 & 70,00 & 74,00 & 77,00 \\
\hline
\end{tabular}


GAHONA, G. O.; GRANIC, M. X.; ANTÚNEZ, CH. M. C.; ARGANDOÑA, P. J.; DE LA FUENTE, Á. M.; DOMANCIC, A. S. \& DÍAZ-NARVÁEZ, V. Evaluación y comparación de la estabilidad de implantes dentales en el maxilar y la mandibula en tres tiempos distintos, mediante análisis de frecuencia de resonancia. Int. J. Odontostomat., 10(3):475-481, 2016.

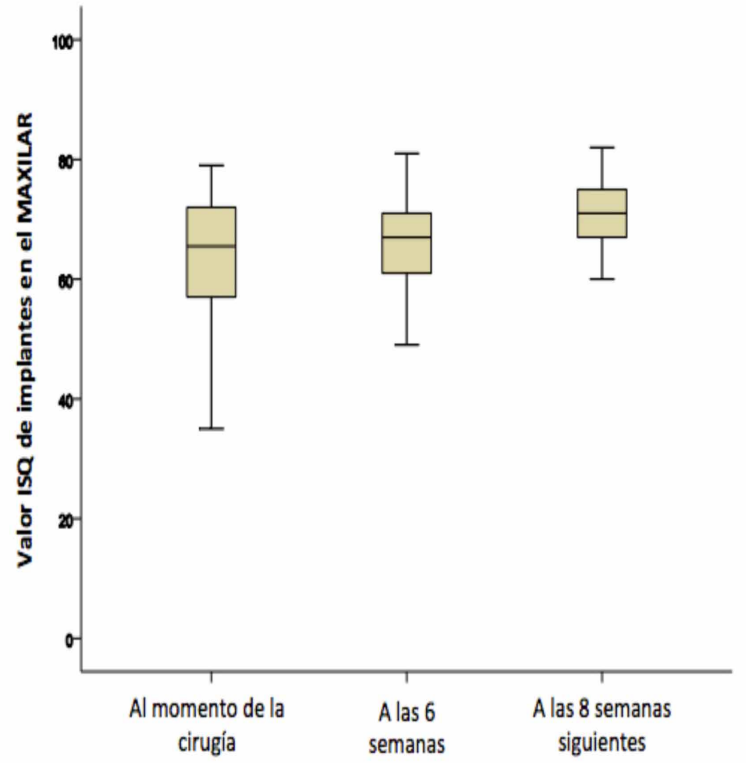

Fig. 1. Gráfico de cajas con las medianas de las mediciones del valor ISQ de los implantes instalado en el maxilar al momento de la cirugía, a las 6 semanas y a los 2 meses.

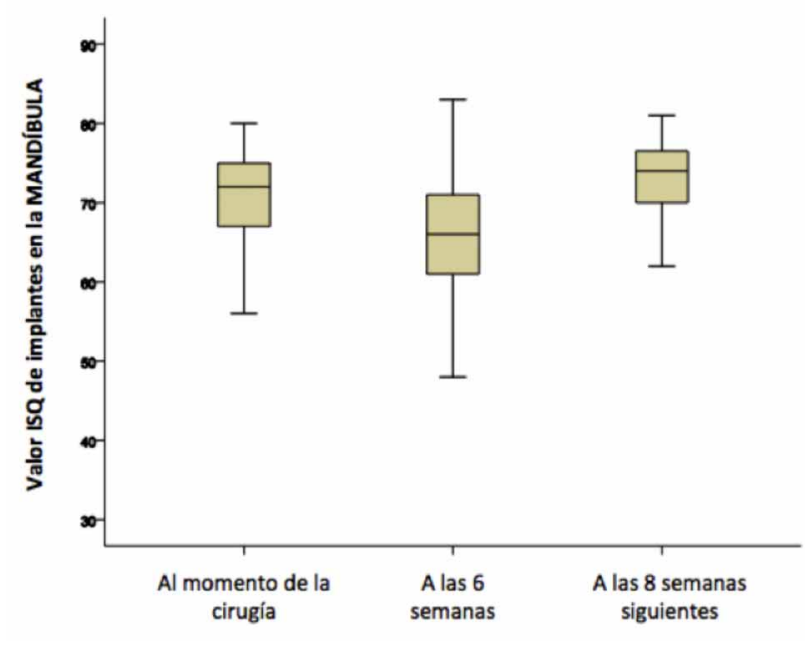

Fig. 2. Gráfico de cajas con las medianas de las mediciones del valor ISQ en la mandíbula al momento de la cirugía, a las 6 semanas y a los 2 meses.

Tabla V: Resultados de la comparación entre las medianas de los ISQ obtenidos en el maxilar y la mandíbula en los tres tiempos examinados.

\begin{tabular}{|c|c|c|c|c|}
\hline & & $\begin{array}{l}\text { Valores ISQ en el } \\
\text { maxilar al momento de la } \\
\text { cirugía / Valores ISQ en } \\
\text { la mandibular al } \\
\text { momento de la cirugía. }\end{array}$ & $\begin{array}{c}\text { Valores ISQ en el maxilar a } \\
\text { las } 6 \text { semanas / Valores } \\
\text { ISQ en la mandibular a las } \\
6 \text { semanas. }\end{array}$ & $\begin{array}{c}\text { Valores ISQ en el } \\
\text { maxilar a los } 2 \\
\text { meses / Valores ISQ } \\
\text { en la mandibular a } \\
\text { los } 2 \text { meses. }\end{array}$ \\
\hline Diferencias más extremas & Absoluta & ,252 & ,107 &, 186 \\
\hline & $\begin{array}{l}\text { Positiva } \\
\text { Neqativa }\end{array}$ & $\begin{array}{l}, 000 \\
-.252\end{array}$ & $\begin{array}{l}, 107 \\
-105\end{array}$ & $\begin{array}{r}, 020 \\
-186\end{array}$ \\
\hline Z de Kolmogorov-Smirnov & & 1,281 &, 544 &, 944 \\
\hline Sig. asintót. (bilateral) & &, 075 & ,929 & 335 \\
\hline Sig. exacta (bilateral) & &, 040 & ,756 & 190 \\
\hline
\end{tabular}

\section{DISCUSIÓN}

El éxito a largo plazo de los implantes dentales, depende de la calidad de la interfase hueso-implante. La estabilidad primaria es clave en la sobrevida de los implantes, entre los factores que la determinan es de gran importancia la calidad del tejido óseo (Albrektsson et al., 1981).

La pared cortical oclusal, donde se fresa para la instalación del implante, posee un grosor de 0,5 a 2,5 mm. Representando aproximadamente un $10 \%$ del largo del implante (Katranji et al., 2007). Pero bajo de esta, la arquitectura ósea puede poseer un mayor o menor componente trabecular. El maxilar se caracteriza por poseer mayor componente trabecular, a diferencia de la mandíbula, la cual posee mayor densidad ósea tanto a nivel trabecular como cortical (Park et al., 2008).

Makary et al. (2012) estudió el comportamiento de la estabilidad de implantes en maxilar y mandíbula mediante volumen óseo, torque de inserción e ISQ al momento de la cirugía, a las 3 y a las 6 semanas post 
GAHONA, G. O.; GRANIC, M. X.; ANTÚNEZ, CH. M. C.; ARGANDOÑA, P. J.; DE LA FUENTE, Á. M.; DOMANCIC, A. S. \& DÍAZ-NARVÁEZ, V. Evaluación y comparación de la estabilidad de implantes dentales en el maxilar y la mandíbula en tres tiempos distintos, mediante análisis de frecuencia de resonancia. Int. J. Odontostomat., 10(3):475-481, 2016.

cirugía. Aunque los valores ISQ registrados por ellos fueron más altos, a diferencia de nuestro estudio, no encontraron diferencias significativas entre ambas arcadas durante los tiempos estudiados y tampoco en el maxilar y la mandíbula por separados. Si encontraron asociación entre volumen óseo y valor ISQ. Existe una serie de factores que pueden influenciar la estabilidad de los implantes al momento de la cirugía (estabilidad primaria) como son la densidad ósea, la geometría del implante, la técnica quirúrgica, micro movimientos durante la cicatrización y las características de la superficie del implante. Durante la estabilidad secundaria, el factor más importante que puede afectarla es la calidad ósea (Raghavendra et al. \& Manzano-Moreno et al. 2015). Esta serie de factores pudo haber llevado a conclusiones distintas entre ambos estudios, por lo que es necesario tenerlos en cuenta para cada caso en particular.

Friberg et al. concluyeron que en hueso más blando como lo es el maxilar, la estabilidad de los implantes va aumentando en el tiempo. También se ha estudiado la estabilidad primaria y secundaria en implantes en la mandíbula mediante ISQ. Ellos observaron a la quinta semana el menor valor ISQ, el cual fue aumentando posteriormente (Shokri \& Daraeighadikolaei et al. 2013) En ambos estudios, las conclusiones obtenidas, fueron similares a nuestros resultados.

La reparación ósea es distinta entre el hueso con mayor componente trabecular (maxilar) y el con mayor componente cortical como el mandibular, reflejando factores evolutivos y funcionales. La cicatrización cortical se basa en la remodelación osteonal, mientras que la trabecular se realiza mediante osteogénesis de contacto como resultado de la osteoconducción y la formación de hueso de nuevo (Davies). Esta característica del hueso trabecular, mal llamado de "mala calidad" lo hace adaptarse mucho mejor al proceso de cicatrización que el hueso cortical y podría explicar el por qué la estabilidad de los implantes en el maxilar, pese a ser menor que en la mandíbula, tiene una curva de aumento continua en el tiempo estudiado.

\section{CONCLUSIONES}

Según los resultados de nuestro estudio, es posible concluir que en el maxilar, la estabilidad de los implantes evaluada mediante RFA aumenta continua- mente después de la cirugía de implantes. En la mandíbula, disminuye en la sexta semana y aumenta en las 8 siguientes, alcanzando valores similares a los iniciales. Al comparar La estabilidad de los implantes en maxilar versus mandíbula, es mayor en la mandíbula, solamente al momento de la cirugía. A las 6 y 8 semanas, la estabilidad alcanzada por los implantes tanto en el maxilar como la mandíbula, es similar.

GAHONA, G. O.; GRANIC, M. X.; ANTÚNEZ, CH. M. C.; ARGANDOÑA, P. J.; DE LA FUENTE, Á. M.; DOMANCIC, A. S. \& DÍAZ-narváez, V. Comparison of dental implants stability in maxilla and mandible at three different times using resonance frequency analysis. Int. J. Odontostomat. 10(3):475-481, 2016.

ABSTRACT: The aim of this study was to test and compare dental implant stability placed in the maxilla and the mandible during three time periods, by resonance frequency analysis (RFA). Using a non experimental prospective study we evaluated 103 3i Biomet Osseotite Implants in 90 patients (50 in maxilla and 53 in mandible). An electronic RFA device (Osstell Mentor $^{\circledR}$ ) was used to record the Implant Stability Quotient (ISQ values) at implant surgery, at six weeks and at eight weeks. Concerning the maxilla, median values increase continuously after implant placement. In the mandible, median values seem to be similar in mandibular at the first and the third evaluation time, but decrease in the second one. Separately for both maxilla and mandible, the median values were highly significant, indicating that these differ in the three time periods studied $(p<0.001)$. Significant difference between the median of the maxilla and mandible was found only at the time of implant placement. At 6 and 8 weeks, no significant differences were observed ( $p>0.05)$. In the maxilla, the initial stability of implants is increased in time. In the mandible, initial stability decreases at 6 weeks but increases again at 8 weeks, reaching values similar to the initial. The implant stability was higher in mandible than maxilla only at implant placement. At 8 weeks the stability achieved by the implants in the maxilla and mandible was similar.

KEY WORDS: dental implants, maxilla, mandible, resonance frequency analysis.

\section{REFERENCIAS BILIOGRÁFICAS}

Albrektsson, T.; Brånemark, P. I.; Hansson, H. A. \& Lindstrom, J. Osseointegrated titanium implants. Requirements for ensuring a long-lasting, direct bone-to-implant anchorage in man. Acta Ortho. Scand., 52(2):155-70, 1981.

Atsumi, M.; Park, S. H. \& Wang, H. L. Methods used to assess implant stability: current status. Int. J. Oral Maxillofac. Implants., 22(5):743-54, 2007. 
GAHONA, G. O.; GRANIC, M. X.; ANTÚNEZ, CH. M. C.; ARGANDOÑA, P. J.; DE LA FUENTE, Á. M.; DOMANCIC, A. S. \& DÍAZ-NARVÁEZ, V. Evaluación y comparación de la estabilidad de implantes dentales en el maxilar y la mandíbula en tres tiempos distintos, mediante análisis de frecuencia de resonancia. Int. J. Odontostomat., 10(3):475-481, 2016.

Davies, J. E. Understanding peri-implant endosseous healing. J. Dent. Educ., 67(8):932-49, 2003.

Degidi, M.; Daprile, G. \& Piattelli, A. Determination of primary stability: a comparison of the surgeon's perception and objective measurements. Int. J. Oral Maxillofac. Implants, 25(3):558-61, 2010.

Díaz, N. V. P. Metodología de la Investigación Científica y Bioestadística para profesionales y Estudiantes de Ciencias de la Salud. Santiago de Chile, Ril Editores, 2009.

Díaz, N. V. P. Errores estadísticos frecuentes al comparar dos poblaciones independientes. Rev. Chil. Nutr., 36(4):1136-8, 2009.

Friberg, B.; Sennerby, L.; Meredith, N. \& Lekholm, U. A comparison between cutting torque and resonance frequency measurements of maxillary implants. A 20month clinical study. Int. J Oral Maxillofac. Surg., 28(4):297-303, 1999.

Herrero-Climent, M.; Albertini, M.; Rios-Santos, J. V.; LázaroCalvo, P.; Fernández-Palacín, A. \& Bullon, P. Resonance frequency analysis-reliability in third generation instruments: Osstell mentor®. Med. Oral Patol. Oral Cir. Bucal, 17(5):e801-6, 2012.

Katranji, A.; Misch, K. \& Wang, H. L. Cortical bone thickness in dentate and edentulous human cadavers. J. Periodontol., 78(5):874-8, 2007.

Koh, R. U.; Rudek, I. \& Wang, H. L. Immediate implant placement: positives and negatives. Implant Dent., 19(2):98-108, 2010.

Makary, C.; Rebaudi, A.; Sammartino, G. \& Naaman, N. Implant primary stability determined by resonance frequency analysis: correlation with insertion torque, histologic bone volume, and torsional stability at 6 weeks. Implant Dent., 21(6):474-80, 2012.

Manzano-Moreno, F. J.; Herrera-Briones, F. J.; Bassam, T.; Vallecillo-Capilla, M. F. \& Reyes-Botella, C. Factors affecting dental implant stability measured using the ostell mentor device: A systematic review. Implant Dent., 24(5):565-77, 2015.

Meredith, N.; Alleyne, D. \& Cawley, P. Quantitative determination of the stability of the implant-tissue interface using resonance frequency analysis. Clin. Oral Implants Res., 7(3):261-7, 1996.

Meredith, N.; Book, K.; Friberg, B.; Jemt, T. \& Sennerby, L. Resonance frequency measurements of implant stability in vivo. A cross-sectional and longitudinal study of resonance frequency measurements on implants in the edentulous and partially dentate maxilla. Clin. Oral Implants Res., 8(3):226-33, 1997.
Park, H. S.; Lee, Y. J.; Jeong, S. H. \& Kwon, T. G. Density of the alveolar and basal bones of the maxilla and the mandible. Am. J. Orthod. Dentofacial Orthop., 133(1):307, 2008.

Raghavendra, S.; Wood, M. \& Taylor, T. D. Early wound healing around endosseous implants: a review of the literature. Int. J. Oral Maxillofac. Implants, 20(3):425-31, 2005.

Shokri, M. \& Daraeighadikolaei, A. Measurement of primary and secondary stability of dental implants by resonance frequency analysis method in mandible. Int. J. Dent., 2013:506968, 2013.

Siegel, S. \& Castellan, N. J. Nonparametric Statistics for the Behavioral Sciences. New York, McGraw-Hill, 1988.

Dirección para correspondencia:

Dr. Osvaldo Gahona Gutiérrez.

Avenida Suecia 1482, Depto. 706

Providencia

Santiago

CHILE

Correo: ogahona@odontologia.uchile.cl

Received: 08-09-2016

Accepted: 17-10-2016 